\title{
DEVELOPMENT OF AN INERTIAL TRACK GEOMETRY MEASURING TROLLEY AND UTILIZATION OF ITS HIGH- PRECISION DATA
}

\author{
YASUKUNI NAGANUMA ${ }^{1}$, TARO YADA ${ }^{2} \&$ TAKAYUKI UEMATSU ${ }^{2}$ \\ ${ }^{1}$ Central Japan Railway Company, General Technology Division, Komaki, Japan \\ ${ }^{2}$ Central Japan Railway Company, Shinkansen Operations Division, Tokyo, Japan
}

\begin{abstract}
In 2015, a truly lightweight track geometry measuring trolley was successfully developed to enable real-time measurement after all track maintenance work. A commercial product model named the LRS100 was realized that is small (disassembled total length of 1,600 mm maximum) and lightweight (13.4 kg, which is about one-third that of the track geometry measuring trolley the LIGHTREC). Seven items may be measured in real-time: track gauge, cross level, alignment (right and left), longitudinal level (right and left), and twist. The measuring accuracy is high, and it far outperforms current track geometry measuring trolleys in terms of repeatability error. The mechanism of the LR-S100 is very simple and easy to maintain because there are no moving parts for measuring alignment and longitudinal level. These advantages, which are not found in any similar conventional product, were achieved with a brand-new track geometry measuring principle called the 'differential-difference method' for the LR-S100. Since the measurement accuracy of the LR-S100 is high, it is also possible to restore the actual track geometry and to compensate for errors in gauge and cross-level irregularities by means of a three-wheel trolley without any additional sensors.
\end{abstract}

Keywords: actual track geometry, D1, Kalman filter, MEMS gyroscope, track geometry measuring trolley, versine.

\section{INTRODUCTION}

As part of maintenance planning at JR-Central, track geometry is measured by a high-speed track recording car every 10 days on the Tokaido Shinkansen [1] and twice a month on conventional lines. To confirm the state of the track after maintenance work, a measuring system built into the maintenance machine is frequently used after ballast renewal work, and a track geometry measuring trolley known as the LIGHTREC [2] is used after concrete sleeper renewal. However, even today, track that is maintained manually is still measured by means of a manual survey using a chord and scale. Many types of portable measuring equipment and trolleys, including the LIGHTREC, have been developed and sold globally. Nevertheless, these are rarely used after manual work, because most of the equipment is too large and heavy to transport and remove from the track when a train passes. Moreover, it is too expensive to purchase the number of devices required for every maintenance site. To provide a solution to such problems, in 2015, we once again launched the development of a small, inexpensive and lightweight track geometry measuring trolley that utilizes the latest sensors and data processing technology, and a new measurement trolley named the LR-S100 was successfully developed [3].

In this paper, a brand-new track geometry measurement method developed for the LR-S100 named the 'differential-difference method' and the computation procedures to convert the DD-data into conventional track parameters such as versine and D1 track irregularity prescribed in EN13848 are described. Furthermore, two examples of utilization of the LR-S100 measurement data are introduced. The first is restoration of the actual track geometry on the ground. The versine data provides sufficient information to maintain running safety and good 
riding comfort, the waveform is different from the actual track geometry. In order to properly manage the track shape and further improve riding comfort, it is necessary to back-calculate the actual track geometry from track irregularity data. Also, actual track geometry is indispensable as input data for the vehicle dynamics simulator. Therefore, we developed actual track geometry restoration techniques that use the Kalman filter and a particle filter. Because the LR-S100 is much more accurate than conventional trolleys, there is a possibility that the actual track geometry can be restored accurately. The second application is to propose a method to compensate for the gauge and cross-level measured by a three-wheel inertial trolley like the LR-S100. Sometimes cost, space or other restrictions do not allow the sensor to be mounted in the proper position on a track geometry measuring trolley, which is required to be small and lightweight, and the measurement results are approximate values. For example, with a three-wheel inertial trolley, the gauge and cross-level are unable to be measured at the same corresponding points on the left and right rails [4]. By recalculating the gauge and cross-level from the restored actual track geometry, the correct value can be estimated.

\section{CONVENTIONAL MEASUREMENT PRINCIPAL}

\subsection{Second-order difference method}

Most conventional equipment and trolleys adopt the second-order difference method. Since this measures track geometry by detecting the relative displacement of three points, it has also been called the three-point method.

Figure 1(a) shows a typical setup for the measurement of longitudinal level or alignment using the second-order difference method. A stiff beam (about 1.0-3.0 m), which is the measuring standard, is set along a rail, and the relative distance between the centre point of the beam and rail is measured using a displacement gauge. The measurement beam is required to have a sufficiently high rigidity, because even a slight deflection of the beam results in a large error in the measurement. This beam requirement has prevented the miniaturization as well as weight and cost reduction for portable measuring equipment. Fig. 1(b) shows another setup for the second-order difference method, which has been devised for LIGHTREC.

\subsection{First-order difference method}

A few types of track geometry measuring trolleys do not adopt the second-order difference method. In one type, a trolley detects the slope of the track (pitch angle of the trolley) using an inclinometer, which is equipped for standard measuring, and then the longitudinal level is obtained by integrating the slope over the distance [5]. This method measures the difference

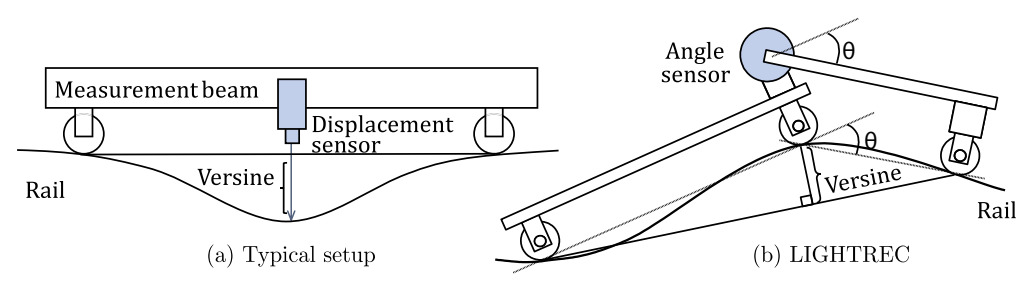

Figure 1: Example of setup for second-order difference measurement. 
between two points of the track geometry, so it is called the first-order difference method or the two-point method. Since the first-order difference method requires two measuring points, it is an attractive method for measuring track geometry. However, it is difficult to apply for alignment, because an expensive device such as an INS (Inertial Navigation System) or an AHRS (Attitude Heading Reference System) is required to detect the yaw angle.

\section{NEW MEASUREMENT METHOD FOR LR-S100}

\subsection{Two-point angle method}

For high accuracy profile measurements, a measuring method known as the two-point angle method is frequently used [6]. Figure 2 shows a schematic of the principle behind this method. Two optical sensors, known as a laser autocollimator, are mounted on a beam to detect local gradients of the profile of the measured object. A difference operation for the two sensors can be used to cancel errors due to pitching and bouncing motion of the measurement beam. When the profile of the object is $x(\xi)$ and the distance of the sensor is $L$, the measurement signal of the two-point angle method is expressed by eqn (1).

$$
r(\xi)=\frac{d}{d \xi}(x(\xi+L)-x(\xi))
$$

Just as with the first-order difference method, the two-point angle method requires only two measurement points, so it presents a highly attractive method for measuring track geometry. However, because the laser autocollimator is affected by the reflection factor of the measurement object, it is difficult to apply this method to track geometry measurement because rails are not ideal surfaces for an autocollimator as reflected light is dampened due to grime and reflected fully at wheel contact points.

\subsection{Differential difference method}

The above two-point method was modified and adopted as the measuring principle for a new track geometry measuring trolley. If angular velocity, which is a time derivative, is converted into an inclination, which is a distance derivative, the two-point angle method is applicable to track geometry measurement using only an inexpensive MEMS (Micro-Electro Mechanical Systems) gyroscope instead of expensive INS or autocollimator. Based on this idea, a method which uses a gyroscope to obtain the same result as the two-point angle method is illustrated

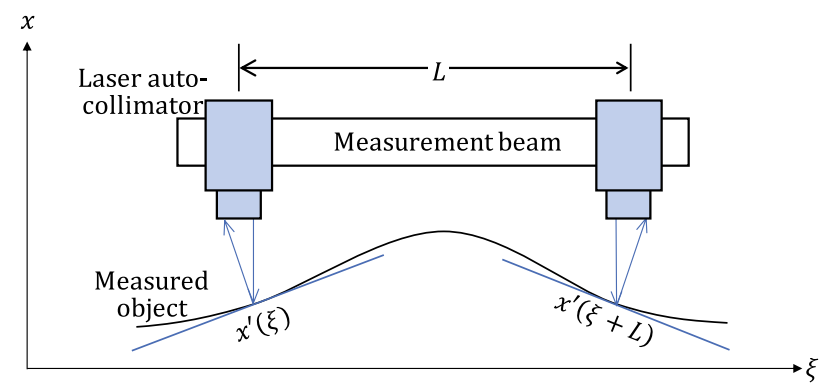

Figure 2: Principle of the two-point angle method (moving laser autocollimator). 


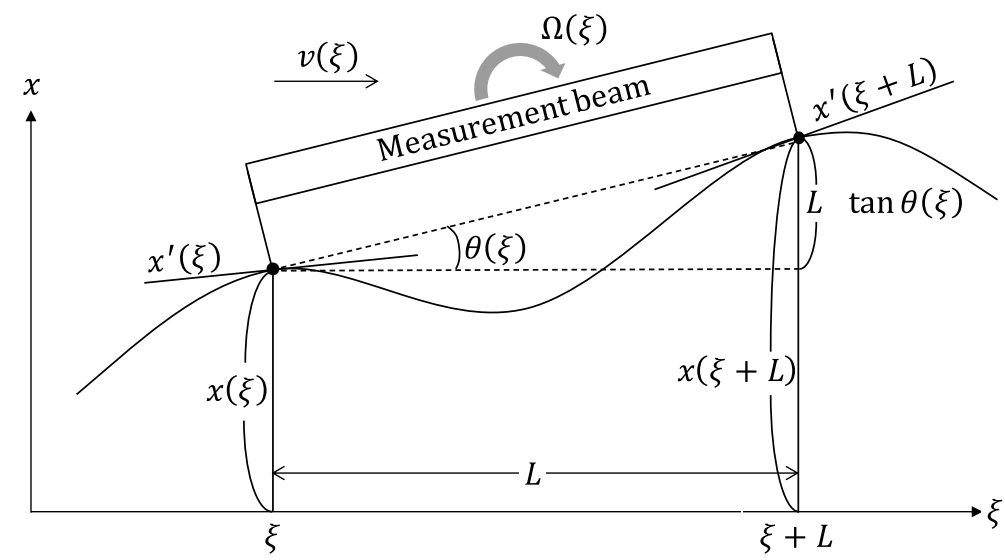

Figure 3: Principle of the differential difference method.

in Fig. 3. The difference in the local gradient $(r(\xi))$ is obtained by eqn (2) using the angular velocity $\Omega(\xi)$ measured by the gyroscope.

$$
\begin{aligned}
r(\xi) & =\frac{d}{d \xi}(x(\xi+L)-x(\xi))=\frac{d}{d \xi}(L \cdot \tan \theta(\xi)) \\
& =L \underbrace{\frac{1}{\cos ^{2} \theta(\xi)}}_{\cong 1.0} \cdot \underbrace{\frac{d}{d t} \theta(\xi)}_{\Omega(\xi)} \cdot \underbrace{\frac{d t}{d \xi} \cong \frac{L \cdot \Omega(\xi)}{v(\xi)},}_{1 / v(\xi)}
\end{aligned}
$$

where $x(\xi)$ is the actual track geometry on the ground, $L$ is the length of the measurement beam. By putting $d \theta(\xi) / d t=\Omega(\xi)$ (angler velocity), $d \xi / d t=v(\xi)$ (measuring velocity) and introducing approximation of $\cos ^{2} \theta(\xi) \cong 1.0$ because $\theta$ is relatively small, $r(\xi)$ can be obtained with extremely simple calculation. We named this newly devised track measuring principle the 'differential difference method (DD-method)' [3].

Eqn (2) may be re-expressed in eqn (3) when $L=2 l$.

$$
r(\xi)=\frac{d}{d \xi}(x(\xi+l)-x(\xi-l)) \text {. }
$$

The frequency response function $H(\omega)$ is expressed as eqn (4) using the Fourier transform of eqn (3).

$$
H(\omega)=j \omega\left(e^{j \omega l}-e^{-j \omega l}\right)=-2 \omega \sin \omega l,
$$

where $\omega(=2 \pi f)$ is the angular frequency and $f$ is the spatial frequency. Gain and phase characteristics are expressed as shown in eqns (5) and (6).

$$
\begin{aligned}
& |H(\omega)|=2 \omega|\sin \omega l| \\
& \theta(\omega)=0
\end{aligned}
$$




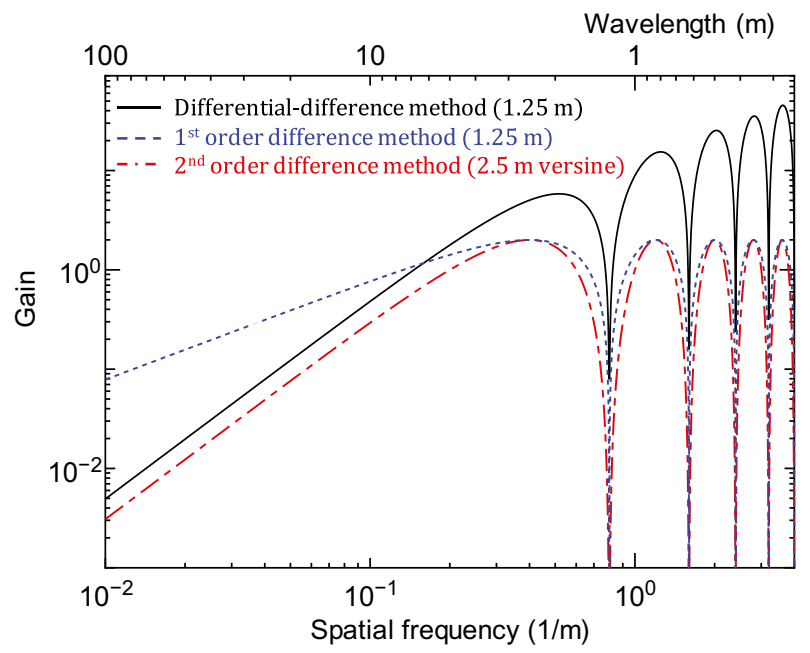

Figure 4: Gain characteristics of the DD-method.

The gain of the DD-method and conventional methods are compared in Fig. 4. Since its gain in a short wavelength range is relatively high, the DD-method may diagnose the short wavelength rail roughness at the same time as it measures track irregularity.

Figure 5 shows a new track geometry measuring trolley named LR-S100 which adopts the DD-method. The weight of the body (excluding a push-pull bar and display unit) is a very

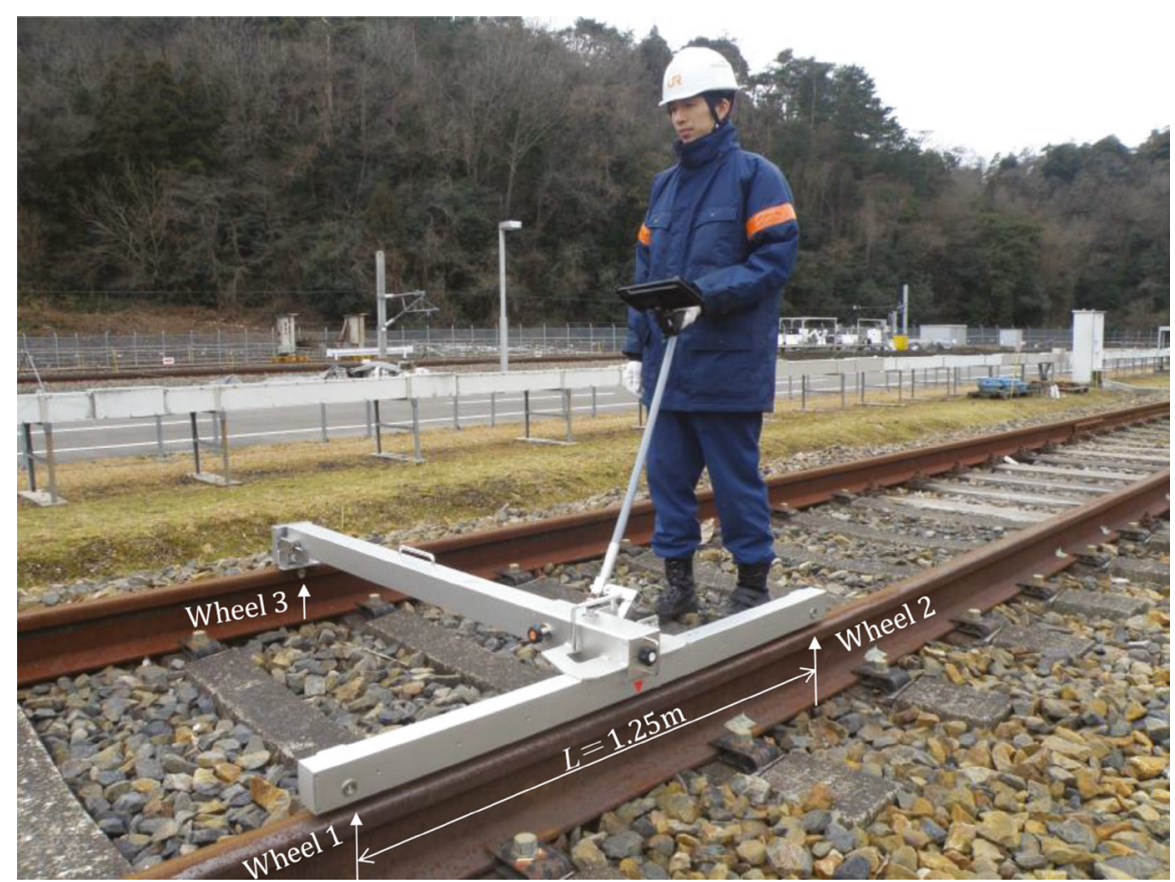

Figure 5: The LR-S100 inertial trolley adopting the DD-method. 
light at $13.4 \mathrm{~kg}$. The measurement rail side requires only two wheels. Furthermore, there are no mechanical moving parts for measuring alignment, longitudinal level and cross level. All these features have been achieved by the adoption of the DD-method.

\section{CONVERSION TO TRACK IRREGULARITY PARAMETERS}

\subsection{Versine}

The $10 \mathrm{~m}$ versine method has the advantages of extracting the track geometry of the wavelength of about 10-20 m corresponding to the natural frequency of the truck and being easy to measure after maintenance work, so many track engineers use versine as the track management parameter. Therefore, the DD-signal must be converted to a versine. In this section, the conversion procedure to versine is explained and an effective method is described. The $2.5 \mathrm{~m}$ versine signal $y(\xi)$ may be expressed by following eqn (7):

$$
y(\xi)=x(\xi)-\frac{x(\xi-1.25)-x(\xi+1.25)}{2} .
$$

From eqn (7), a transfer function for a $2.5 \mathrm{~m}$ versine measurement on the $z$-plane yields eqn (8). In this equation, the sampling distance is $0.25 \mathrm{~m}$ with an output delay of $1.25 \mathrm{~m}$ to satisfy the principle of causality.

$$
H_{V S}(z)=-\frac{1}{2}+z^{-5}-z^{-10}=-\frac{1}{2} \underbrace{\left(1-z^{-5}\right)^{2}}_{\text {2nd difference }} .
$$

Eqn (8) shows that a characteristic of the $2.5 \mathrm{~m}$ versine consists of two difference filters and one multiplier [7]. In the same manner as the versine measurement, the transfer function of the DD-method is expressed as eqn (9) from eqn (2).

$$
H_{D D}(z)=\underbrace{\left(1-z^{-1}\right)}_{\text {differential }} \underbrace{\left(1-z^{-5}\right)}_{\text {difference }} .
$$

From eqns (8) and (9), the conversion from DD-signal data to versine is shown in eqn (10).

$$
H_{V S}(z)=-\frac{1}{2} \underbrace{\left(1-z^{-5}\right)}_{\text {difference }} \underbrace{\left(\frac{1}{1-z^{-1}}\right)}_{\text {integral }} \cdot H_{D D}(z) .
$$

Eqn (10) shows that 'after the difference and integration are executed for the DD-data, and the coefficient -0.5 is multiplied, the product is versine'. The block diagram is shown in Fig. 6(a).

Furthermore, the product of the difference $\left(1-z^{-5}\right)$ and integrator $\left(1 / 1-z^{-1}\right)$ becomes a moving summation by polynomial division.

$$
\frac{1-z^{-5}}{1-z^{-1}}=1+z^{-1}+z^{-2}+z^{-3}+z^{-4} .
$$

The above eqn (11) shows that the DD-signal data is completely converted to versine by the simple and stable calculus of moving summation. Figure 6(b) shows a block diagram of the versine conversion algorithm of moving summation. It is notable that measurement by the DD-method and conversion to versine are very simple and may be performed with 


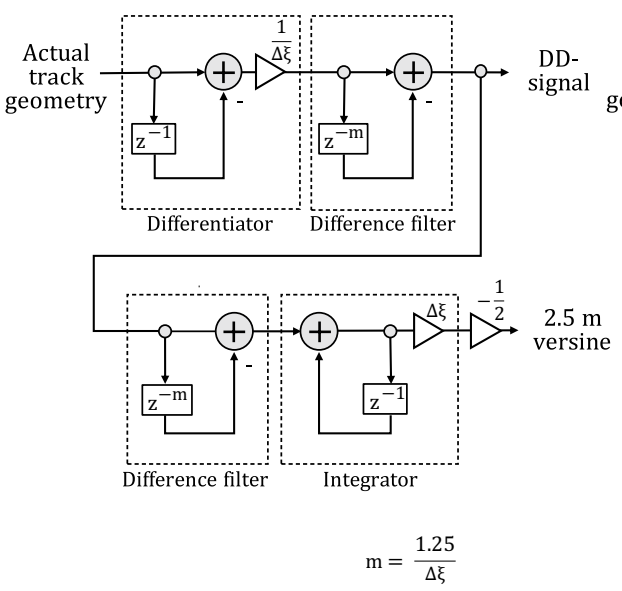

(a) Difference filter and Integrator

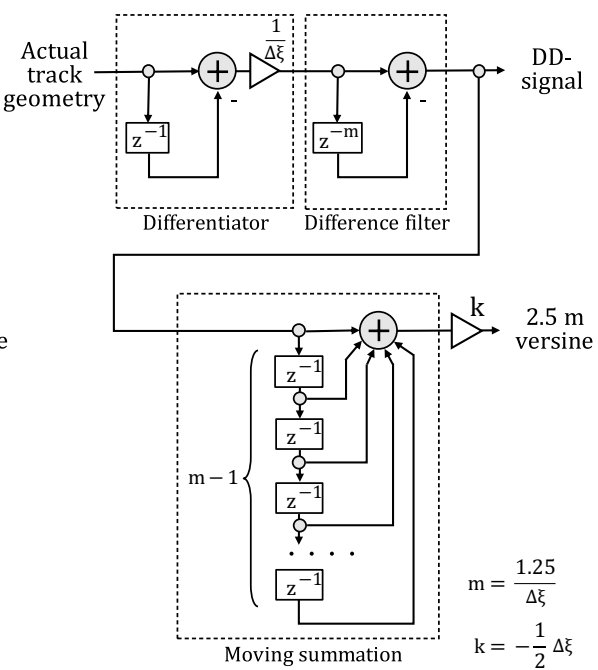

(b) Moving summation

Figure 6: Conversion from DD-signal to versine signal.

minimal operation. Furthermore, the desired chord length versine, such as $10 \mathrm{~m}$, can be easily calculated. The simple data processing contributes to cost reduction and electric power savings for commercialization of the product because the latest CPU (Central Processing Unit) is not necessary.

Figure 7 shows the repeatability of the $10 \mathrm{~m}$ versine alignment calculated from the LR-S100 measurement results. The LR-S100 is a simple structure that is compact, lightweight and does not have a complex mechanism, but it has high repeatability accuracy that surpasses that of conventional track measuring trolleys. As shown in the lower graph of Fig. 7, the difference between the first measurement and the second measurement is within $\pm 0.2 \mathrm{~mm}$.

\subsection{D1 track irregularity (EN13848)}

Because EN13848-4 requires the track irregularity index known as D1 rather than a versine, conversion to the D1 is indispensable for development for a worldwide market. D1 track irregularity is a band-limited actual track geometry on the ground, and the waveband ranges from 3.0 to $25 \mathrm{~m}$. There are two methods for converting to D1: a method calculating from $2.5 \mathrm{~m}$ versine and a method calculating directly from DD-signal data. This paper addresses a re-colouring filter for the DD-signal. The conditions of the designed re-colouring filter are shown in Table 1.

In Fig. 8(a) indicates the impulse response function (filter coefficient), while (b) indicates the frequency response function of the re-colouring filter with DD measurement and the comprehensive frequency response function given as the result of re-colouring. The results of the repeatability tests of D1 signals measured from the LR-S100 are shown in Fig. 9. The maximum repeatability error of the D1 alignment is $0.071 \mathrm{~mm}$, and that of the longitudinal level is $0.062 \mathrm{~mm}$. It was confirmed that the LR-S100 sufficiently satisfies an uncertainty of $\pm 1 \mathrm{~mm}$ as required by EN13848-4. 


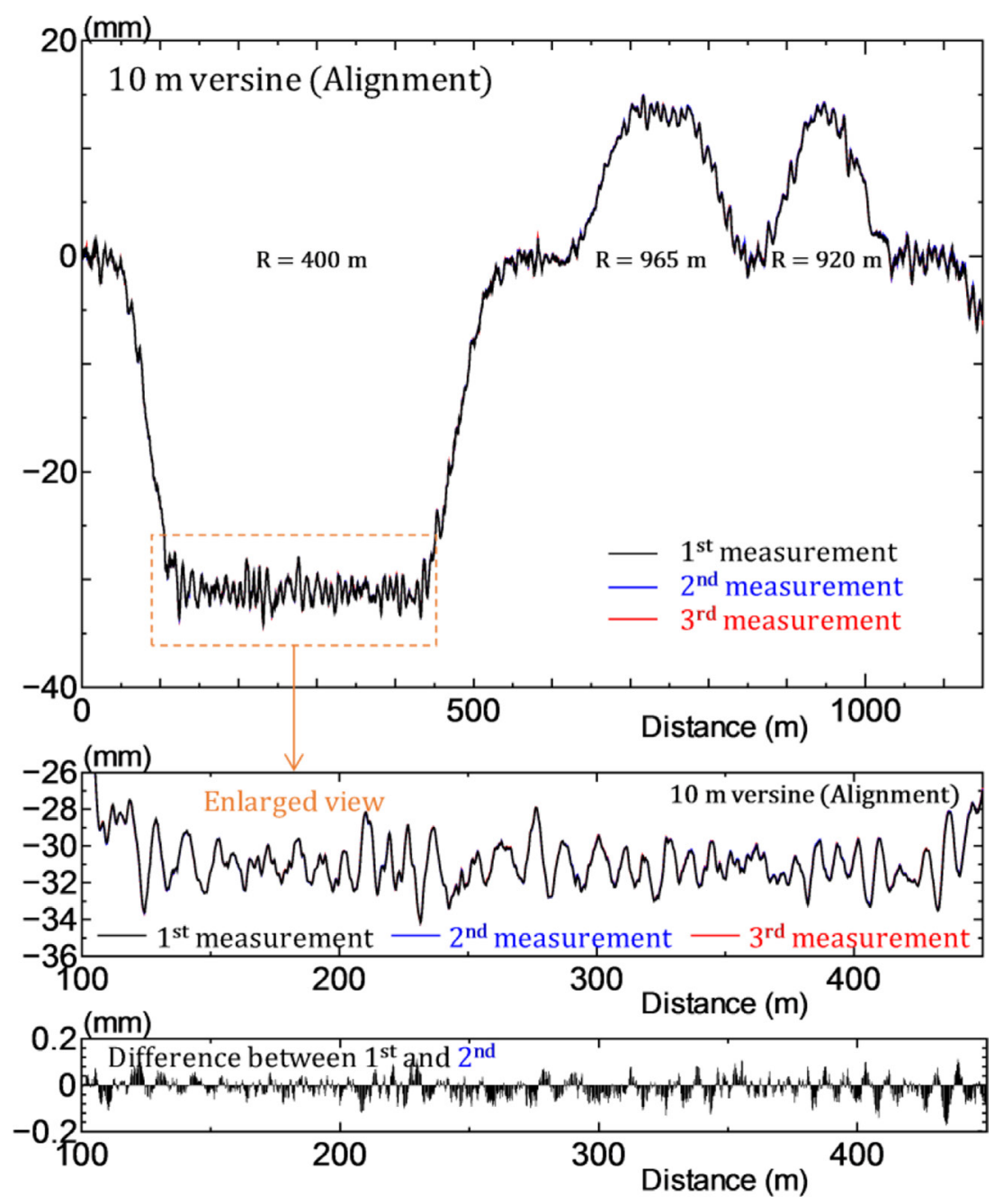

Figure 7: Repeatability of $10 \mathrm{~m}$ versine alignment measured by the LR-S100.

Table 1: Design conditions of the re-colouring filter for DD measurement data.

\begin{tabular}{ll}
\hline Sampling spacing & $0.05 \mathrm{~m}$ \\
\hline Number of impulse responses (number of taps) & 3,001 \\
Pass band & $3-25 \mathrm{~m}$ \\
Transition band (short wavelength) & $1.25-3 \mathrm{~m}$ \\
Transition band (long wavelength) & $25-50 \mathrm{~m}$ \\
Gain of stop band & 0.0001 \\
\hline
\end{tabular}




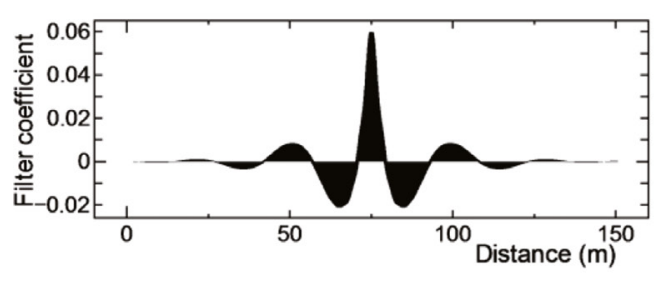

(a) Impulse response functions

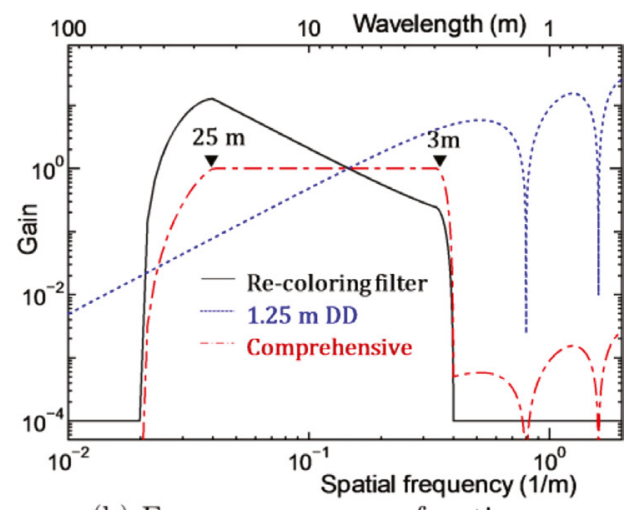

(b) Frequency response functions

Figure 8: Re-colouring filter (from DD signal to D1).

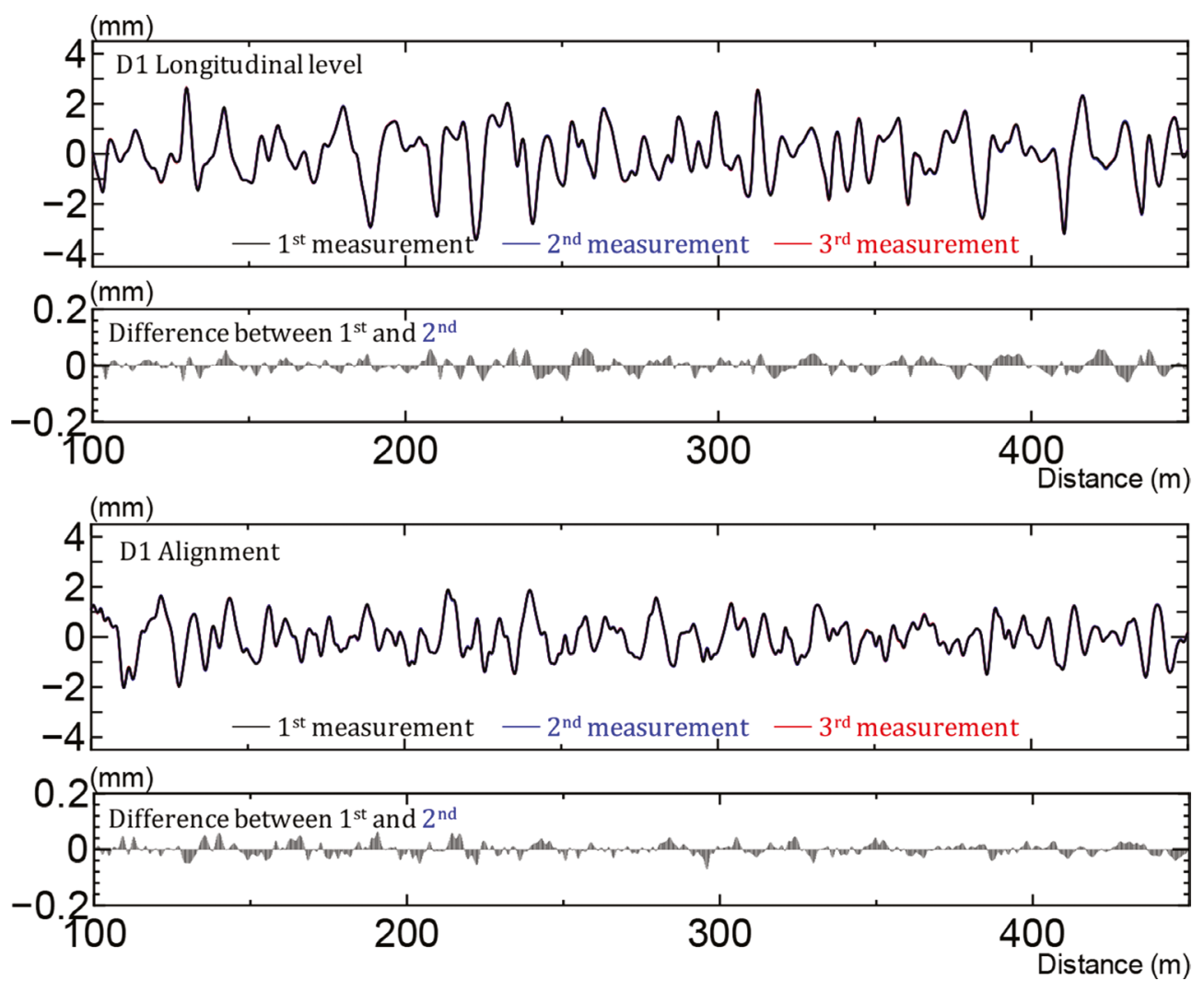

Figure 9: Repeatability of D1 track geometry calculated based on signals measured from the LR-S100. 


\section{APPLICATION OF LR-S100 MEASUREMENT DATA}

\subsection{Restoration of actual track geometry}

In previous studies, we developed a new restoration technique that applies a Bayesian estimation filter to solve the inverse problem of restoring actual track geometry from track irregularity data [8], [9]. When the Kalman filter, one of the most popular Bayesian estimators, is applied, the track irregularity measurements are expressed by linear Gaussian state space models in eqns (12) and (13).

$$
\begin{aligned}
& \boldsymbol{x}_{n}=F \boldsymbol{x}_{n-1}+G \boldsymbol{v}_{n}(\text { system model }), \\
& \boldsymbol{y}_{n}=H \boldsymbol{x}_{n}+\boldsymbol{w}_{n}(\text { observation model }),
\end{aligned}
$$

here, state vector $\boldsymbol{x}_{n}$ which is the actual track geometry, observation vector $\boldsymbol{y}_{n}$ which is the track irregularity measurement data are as following equations.

$$
\boldsymbol{x}_{n}=\left(\begin{array}{llll}
x_{n} & x_{n-1} & \cdots & x_{N}
\end{array}\right)^{T}, \boldsymbol{y}_{n}=\left(\begin{array}{llll}
y_{n} & y_{n-1} & \cdots & y_{N}
\end{array}\right)^{T}
$$

The idea to make the Bayesian estimation filter applicable to the inverse analysis is to use the random walk model or the trend model for the system equation (eqn (12)). We use the second-order trend model $\left(x_{n}=2 x_{n-1}-x_{n-2}+v_{n}\right)$ in this study, so the matrices $F$ and $G$ are as follows:

$$
F=\left(\begin{array}{ccccc}
2 & -1 & 0 & \cdots & 0 \\
1 & 0 & 0 & \cdots & 0 \\
0 & 0 & 0 & \cdots & 0 \\
\vdots & \ddots & \ddots & \ddots & \vdots \\
0 & \cdots & 0 & 1 & 0
\end{array}\right), G=\left(\begin{array}{c}
1 \\
0 \\
0 \\
\vdots \\
0
\end{array}\right) .
$$

$v_{n}$ is the process noise which is assumed to be drawn from a zero mean multivariate normal distribution with covariance $Q$ and $\boldsymbol{w}_{n}$ is the observation noise which is assumed to be zero mean Gaussian white noise with covariance $R$. Since the transfer function of the $2.5 \mathrm{~m}$ versine measurement is eqn (8), so the observation matrix $H$ in eqn (13) is eqn (16) when the data sampling interval is considered to be $0.25 \mathrm{~m}$.

$$
H=\left(\begin{array}{lllllllllll}
-0.5 & 0 & 0 & 0 & 0 & 1 & 0 & 0 & 0 & 0 & -0.5
\end{array}\right)
$$

Inverse analysis of the track geometry using the Kalman filter is enabled based on eqns (17)-(21). In this step of the calculation, the actual track geometry $\boldsymbol{x}_{n}$ is restored as the state variable vector sequentially and stably as follows:

(prediction)

$$
\begin{aligned}
\boldsymbol{x}_{n \mid n-1} & =F \boldsymbol{x}_{n-1 \mid n-1} \\
V_{n-1 \mid n-1} & =F_{n} V_{n-1 \mid n-1} F_{n}^{T}+G_{n} Q_{n} G_{n}^{T}
\end{aligned}
$$


(filtering)

$$
\begin{aligned}
K_{n} & =V_{n \mid n-1} H_{n}^{T}\left(H_{n} V_{n \mid n-1} H_{N}^{T}+R_{n}\right)^{-1} \\
\boldsymbol{x}_{n \mid n} & =\boldsymbol{x}_{n \mid n-1}+K_{n}\left(\boldsymbol{y}_{n}-H_{n} \boldsymbol{x}_{n-1 \mid n-1}\right) \\
V_{n \mid n-1} & =\left(I-K_{n} H_{n}\right) V_{n-1 \mid n-1} .
\end{aligned}
$$

Figure 10 shows the actual track geometry estimated from the $2.5 \mathrm{~m}$ versine alignment using the Kalman filter. The parameters used in the Kalman filter were $\sigma_{v}^{2}=0.02$ and $\sigma_{w}^{2}=1.0 \times 10^{-6}$. Looking at the calculation results, the long wavelength components including the track geometry are restored, which is useful information for maintaining the track shape and further improving riding comfort. In restoring the distance of 1,200 $\mathrm{m}$, the difference between the two results is about $60 \mathrm{~cm}$, and the method can be said to be highly accurate without reference point information. For further utilization in the future such as the construction of new lines, it is better to add information on several reference points.

Figure 11 shows the $10 \mathrm{~m}$ versine alignment recalculated from the restored track geometry. Noteworthy is the fact that the recalculated $10 \mathrm{~m}$ versine, even in the curved sections, matches the measured values very closely. This proves that the restoration result completely retains the information of the measurement data, including long wavelength components. This advantage of the Kalman filter restoration enables various applications in track technology.

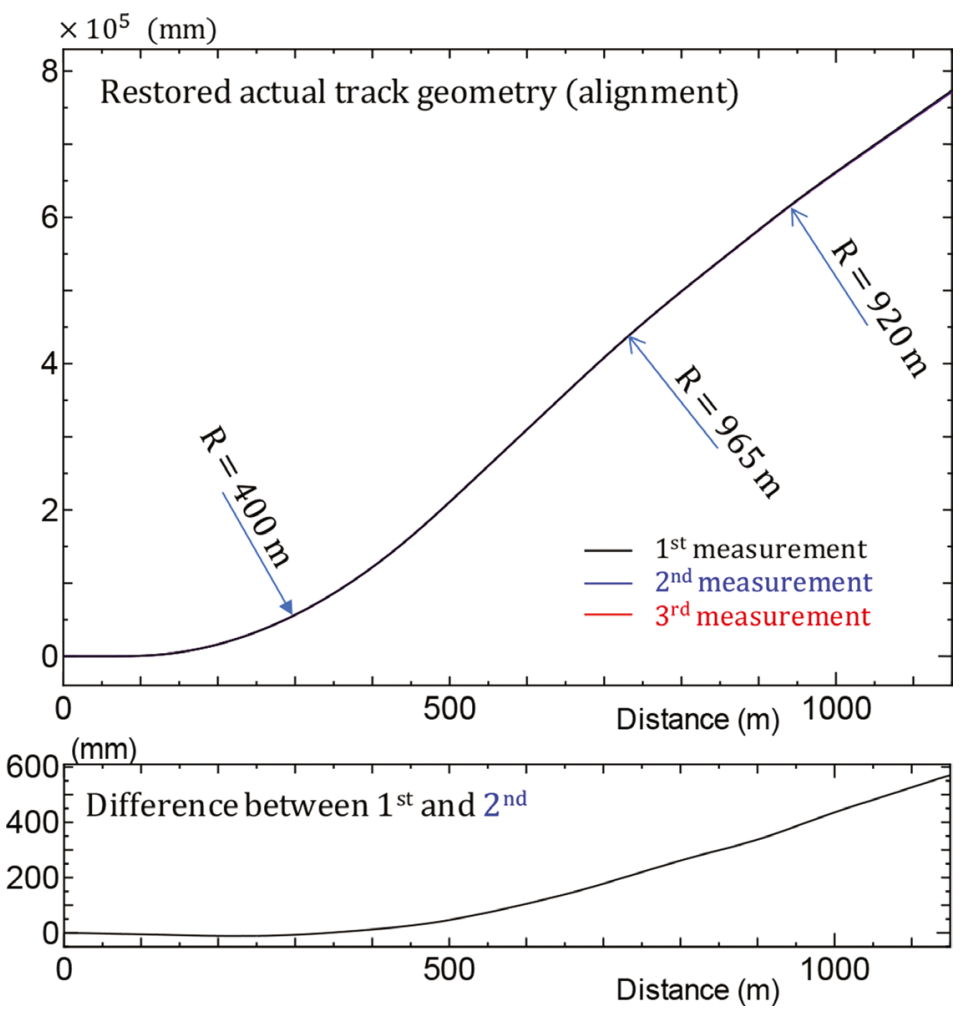

Figure 10: Repeatability of restoration results (alignment). 


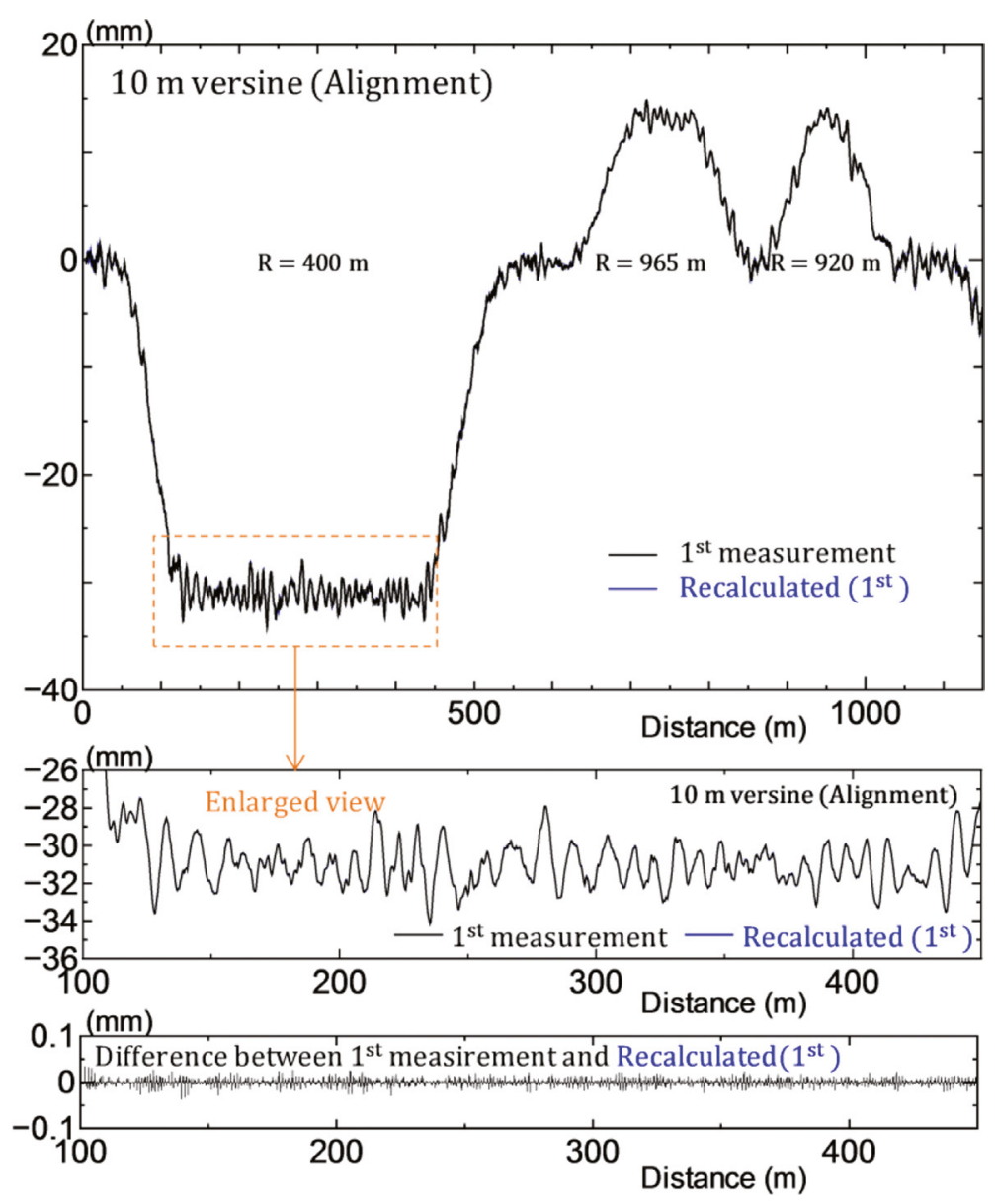

Figure 11: $10 \mathrm{~m}$ versine alignment recalculated from restored actual track geometry.

\subsection{Compensation of a three point gauge and cross-level measured by the LR-S100}

In this section, an example is given of application of the actual track geometry restoration technique described above. This is a method of compensating for gauge and cross-level measured by a three-wheel inertial trolley, such as the LR-S100. Since the LR-S100 has three wheels, the gauge and cross-level are approximate measurements. Figure 12 shows the gauge measurement by the inertial trolley of the three wheel structure. In Fig. 12, the gauge irregularity $g(\xi)$ measured by the three-wheel inertial trolley is expressed in eqn (22).

$$
g(\xi)=\frac{T_{L}(\xi-l)+T_{L}(\xi+l)}{2}-T_{R}(\xi)
$$

Here, $\mathrm{T}_{L}$ is the actual track geometry of the left rail, $\mathrm{T}_{R}$, is that of the right rail, and $l$ is the half length of the trolley measuring beam. The legal gauge $(G)$ is defined in eqn (23).

$$
G(\xi)=T_{L}(\xi)-T_{R}(\xi)
$$




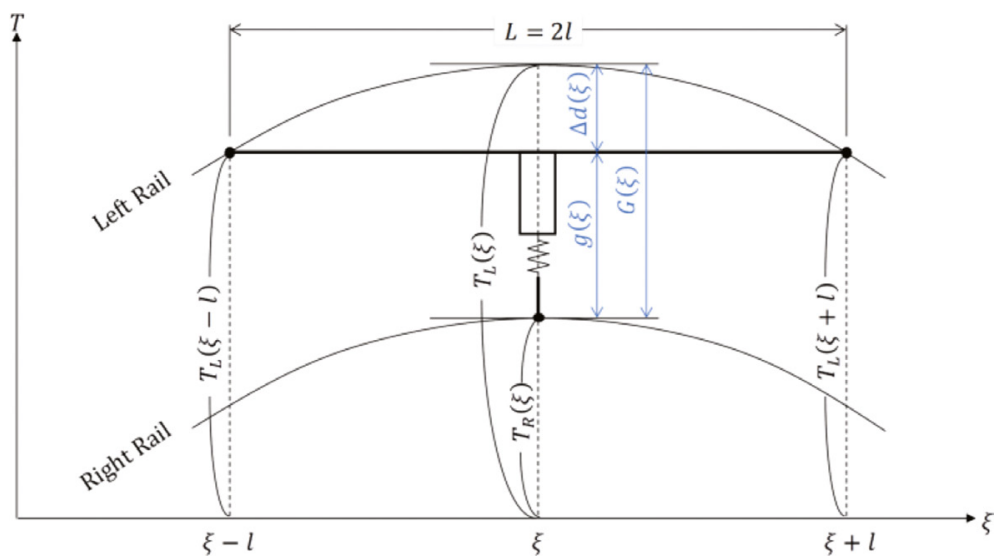

Figure 12: Gauge irregularity measured by a three-wheel inertial trolley.

Therefore, as shown in eqn (24), the difference $\Delta d$ becomes versine, where the length between two wheels is chord length $L$, which is equal to $1.25 \mathrm{~m}$ versine in the LR-S100.

$$
\Delta d(\xi)=G(\xi)-g(\xi)=T_{L}(\xi)-\frac{T_{L}(\xi-l)+T_{L}(\xi+l)}{2} .
$$

This $\Delta d$ is very small when the straight section or the curve radius $\left(R_{c}\right)$ is large, but this difference term is too large to ignore in the case of a subway, tramway or other railway where the curve radius is small. Figure 13 is a comparison between the three points gauge $(g)$ of the LR-S100 and the correct two-point gauge $(G)$ measured by the conventional trolley in the tramway track, and the minimum curve radius of this section is $23 \mathrm{~m}$. The difference may be calculated by the classic formula $\left(L^{2} / 8 R_{c}\right)$ which will also be approximately $8.5 \mathrm{~mm}$. For the LR-S100, $\Delta d$ is estimated by a simple method using $2.5 \mathrm{~m}$ versine alignment, and the gauge is corrected in real time. In this section, a more accurate compensation method that uses the actual track geometry described above was examined. For example, when the actual track geometry $T_{L}$ of the left rail is obtained using the method described in Section 5.1, first the eqn (22) is used to calculate the track geometry $T_{R}$ of the opposite right rail. Next, using eqn (23), we can obtain a correct gauge as defined. Figure 14 shows a comparison of three measurement results of the compensating three-point gauge, using the actual track geometry estimated by the Kalman filter and the two-point gauge measured with a conventional trolley. As is apparent from the figure, gauge irregularity measured with the LR-S100, which is a

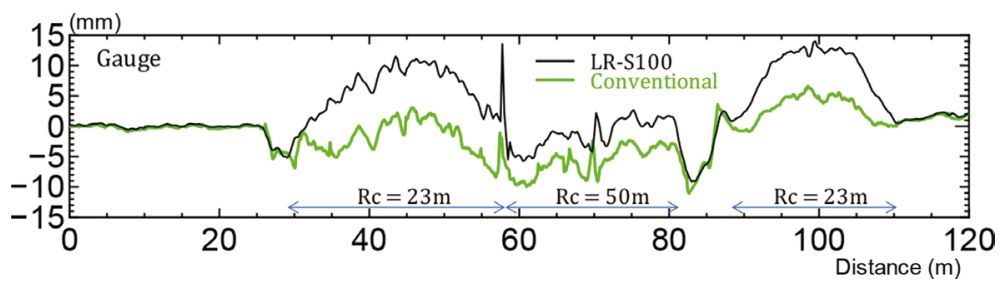

Figure 13: Comparison of three measurements of compensated gauge and gauge measured with conventional trolley. 

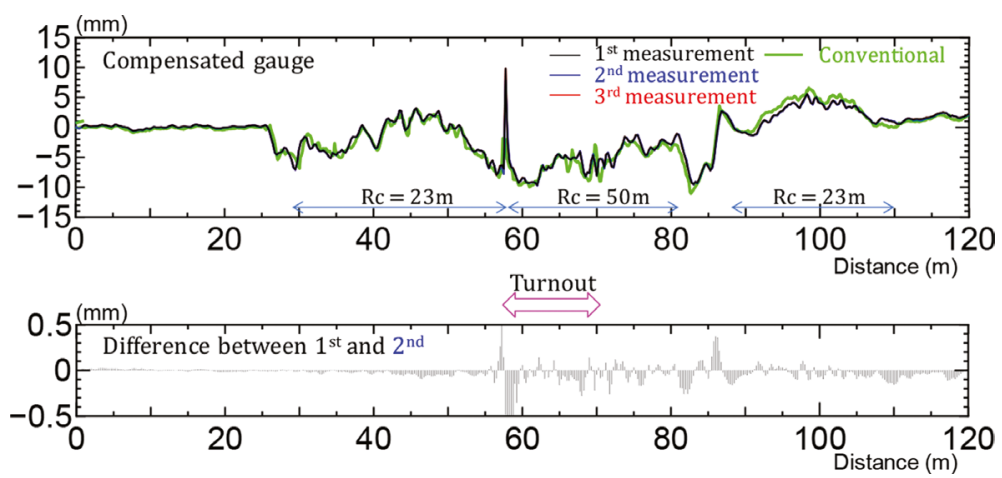

Figure 14: Comparison of gauge irregularity between the LR-S100 and a conventional measuring trolley along a sharp curve.

three-wheel structure, is compensated, and the correct gauge is obtained. Furthermore, except for the turnout section, the difference between the two measurements is within $\pm 0.3 \mathrm{~mm}$, and it is confirmed that the repeatability after the compensation is sufficiently high.

Cross-level irregularity measured by a three-wheel trolley may be compensated in exactly the same manner as gauge irregularity.

\section{CONCLUSION AND FUTURE REMARKS}

The following conclusions were drawn from our development:

(i) In order to check running safety after all track maintenance work, a small, lightweight inertial tack geometry measuring trolley named the LR-S100 was successfully developed. Its total weight is only $13.4 \mathrm{~kg}$.

(ii) On the premise of using the latest high-precision gyroscope, a brand-new track measuring principle named the differential difference method was developed and adopted for the LR-S100. According to the DD-method, stable track irregularity can be obtained by simple calculation using easily measurable signals such as angular rate and travelling speed.

(iii) DD-data can be completely converted to versine by an extremely simple and stable moving sum operation. As a result of the test measurement on the Tokaido Shinkansen line, the repeatability error of $10 \mathrm{~m}$ versine was less than $0.2 \mathrm{~mm}$.

(iv) The DD-signal can also be converted to D1 track irregularity (specified in EN13848) in real time by using the FIR digital filter. The repeatability error of the calculated D1 irregularities was less than $0.1 \mathrm{~mm}$.

(v) A proprietary method applying the state space model and the Kalman filter to inverse analysis may make it possible to restore the actual track geometry of the curved section. A method to compensate for gauge and cross-level irregularities measured by three wheel inertial trolley was proposed. It was confirmed that defined correct gauge can be calculated using actual track geometry restored using the state space model.

Since the LR-S100 is much more accurate than a conventional trolley, various applications such as switch management, surveying at the construction of a new line, and precise track measurement before and after tamping work are expected. 


\section{REFERENCES}

[1] Naganuma, Y., Tanaka, M. \& Ichikawa, K., High-speed track inspection car in the new Dr. Yellow. Proceedings of the World Congress on Railway Research (WCRR 2001), 2001.

[2] Naganuma, Y., Fukushima, S. \& Morimoto, M., Truly portable track recording equipment and its application for track quality management. Proceedings of the World Congress on Railway Research (WCRR 2003), pp. 1313-1323, 2003.

[3] Naganuma, Y. \& Yada, T., Development of truly portable track geometry recording trolley and accompanying new measurement principle. Computers in Railways XV: Railway Engineering Design and Operation, 162(14), pp. 329-342, 2016.

[4] Chen, Q., Niu, X., Zuo, L., Zhang, T., Xiao, F., Liu, Y. \& Liu, J., A railway track geometry measuring trolley system based on aided ins. Sensors, 18(2), p. 538, 2018.

[5] Esveld, C., Modern Railway Track. MRT-Productions, 1989.

[6] Kiyono, S., Kume, T., Asakawa, Y. \& Kamada, O., Differential laser auto-collimation method (2 report). Journal of JSPE, 57(4), p. 652, 1991 (in Japanese).

[7] Naganuma, Y., Kobayashi, M. \& Okumura, T., Inertial measurement processing techniques for track condition monitoring on Shinkansen commercial trains. Journal of Mechanical Systems for Transportation and Logistics, 3(1), pp. 315-325, 2010.

[8] Yoshimura, A. \& Naganuma, Y., Bayesian reconstruction of 3D railway track geometry by particle filter. Proceedings of the Second International Conference on Railway Technology, 2014.

[9] Naganuma, Y. \& Uematsu, T., Reconstruction of actual track geometry using data measured by inertial trolley. WIT Transactions on the Built Environment, WIT Press, 181, 2018. 